\title{
Mielinólise extrapontina secundária à hipernatremia: estudo de caso com desfecho favorável
}

\author{
Extrapontine myelinolysis secondary to hypernatremia: case studyt with favorable outcome \\ Mielinólisis extrapontina secundaria a la hipernatremia: estudo de caso con resultado \\ favorable
}

Adalberto Rosa Neto ${ }^{*}$, Carolina Barros Kahwage ${ }^{2}$, Salomão Georges Kahwage Neto².

\begin{abstract}
RESUMO
Objetivo: Detalhar o caso de uma paciente que evoluiu com mielinólise extrapontina secundária à hipernatremia. Detalhamento do caso: Paciente adulta iniciou quadro de cefaleia unilateral, fraqueza muscular e engasgos constantes progressivos. Após episódio de broncoaspiração de grande quantidade, necessitou intubação orotraqueal. No âmbito hospitalar, detectado sódio sérico de $180 \mathrm{mEq} / \mathrm{L}$ na entrada, que foi corrigido ao longo de 7 dias até sua normalização. $O$ quadro clínico de tetraparesia inicial foi melhorado na medida em que os níveis de sódio normalizavam e uma ressonância magnética de controle mostrou regressão total das lesões. Atualmente, em acompanhamento no regime ambulatorial com poucas sequelas após o ocorrido. Considerações Finais: As síndromes de desmielinização osmótica podem ter apresentações variadas de acordo com o local acometido no encéfalo. Não é possível prever o desfecho, podendo ser totalmente favorável ou catastrófico. Necessitam maior atenção as correções de distúrbios hidroeletrolíticos, pois a prevenção ainda se configura na melhor alternativa para estes pacientes.
\end{abstract}

Palavras-chave: Mielinólise central da ponte, Hipernatremia, Doenças do sistema nervoso.

\begin{abstract}
Objective: To report the case of a patient who developed extrapontine myelinolysis secondary to hypernatremia. Case Detail: Adult patient presented with unilateral headache, muscle weakness, and progressive choking. After an episode of large amount of bronchoaspiration, she required orotracheal intubation. At the hospital, serum sodium of $180 \mathrm{mEq} / \mathrm{L}$ was detected at the entrance, which was corrected over 7 days until normalization. The clinical picture of initial tetraparesis was improved as sodium levels normalized and a control magnetic resonance showed complete regression of the lesions. Currently in outpatient follow-up with few sequelae after the incident. Final considerations: Osmotic demyelination syndromes may have different presentations according to the affected brain location. It is not possible to predict the outcome, which may be totally favorable or catastrophic. More attention needs to be paid on correcting hydroelectrolytic disorders, as prevention is still the best alternative for these patients.
\end{abstract}

Keywords: Central pontine myelinolysis, Hypernatremia, Nervous system diseases.

\section{RESUMEN}

Objetivo: Detallar el caso de um paciente que desarrolló mielinólisis extrapontina secundaria a hipernatremia. Detalle del caso: Paciente adulto con cefalea unilateral, debilidad muscular y asfixia progresiva progresiva. Después de un episodio de gran cantidad de broncoaspiración, el paciente requirió intubación orotraqueal. A nivel hospitalario, se detectó sodio sérico de $180 \mathrm{mEq} / \mathrm{L}$ en la entrada, que se

\footnotetext{
${ }^{1}$ Hospital Ophir Loyola, Belém - PA. *E-mail: adalberto.rosaneto@gmail.com

2 Hospital Jean Bitar, Belém - PA.
} 
corrigió durante 7 días hasta la normalización. El cuadro clínico de la tetraparesia inicial mejoró a medida que los niveles de sodio se normalizaron y una resonancia magnética de control mostró una regresión completa de las lesiones. Actualmente en seguimiento ambulatorio con pocas secuelas después del incidente. Consideraciones finales: Los síndromes de desmielinización osmótica pueden tener diferentes presentaciones según la ubicación del cerebro afectado. No es posible predecir el resultado, que puede ser totalmente favorable o catastrófico. Se debe prestar más atención a la corrección de los trastornos hidroelectrolíticos, ya que la prevención sigue siendo una mejora alternativa para estos pacientes.

Palabras clave: Mielinólisis pontino central, Hipernatremia, Enfermedades del sistema nervioso.

\section{INTRODUÇÃO}

Mielinólise pontina central (MPC) e mielinólise extrapontina (MEP) são doenças que podem ser caracterizadas em conjunto como síndrome de desmielinização osmótica. Ocorrem por desmielinização no encéfalo e podem se localizar na ponte na MPC ou fora dela na MEP. Com o avançar dos estudos e recursos diagnósticos, desde sua primeira descrição, em 1959, foram descobertos grupos de maior vulnerabilidade e particularidades a respeito de fisiopatologia, embora esta ainda não permaneça totalmente esclarecida atualmente (ADAMS RD, et al., 1959).

Acreditou-se, durante muito tempo, que a principal causa de instalação da síndrome fosse a correção rápida de sódio de pacientes hiponatrêmicos e que isso aconteceria especialmente em pacientes com deficiência de vitamina B12. Esta relação foi posteriormente confirmada, porém, existem outras etiologias descritas como base desta desordem encefálica. Dagur G e Khan AS (2015), relatam 10 outras origens para instalação da síndrome, entre elas a hipernatremia, com poucos casos descritos na literatura médica secundária a esta alteração hidroeletrolítica.

Os principais sintomas relacionados às síndromes são tremor, quadriplegia, rebaixamento de nível de consciência, dificuldade de deglutição e fraqueza muscular; porém, há uma outra gama de sintomas neurológicos que podem estar relacionados, a depender de comorbidades de do local afetado pelo encéfalo. A mortalidade é alta e o desfecho imprevisível, podendo apresentar recuperação completa dos sintomas, recuperação parcial ou até levar ao óbito (COÇA KL, et al., 2017; KHAN AS e DAGUR G, 2015; JURNO ME, et al., 2012; BRITO AR, et al., 2006).

A rota mais fácil para suspeição de um caso de mielinólise é através de exame físico e neurológico, que guiará a pesquisa de exames laboratoriais e de imagem. Nos exames laboratoriais, a dosagem de sódio é indispensável. O principal exame de imagem para diagnóstico das síndromes é a ressonância magnética, com achados de hiposinal em T1, hipersinal em T2 e em FLAIR nos locais acometidos (KHAN AS e DAGUR G, 2015; ISMAIL FY, et al., 2013; LAUBENBERGER J, et al. 1996)

Com epidemiologia ainda incerta e diagnóstico difícil de ser dado, acredita-se que a síndrome se apresente em $0,05 \%$ dos pacientes internados em hospitais gerais e de 0,4-0,56\% pacientes internados em serviços de neurologia. É uma doença rara e incomum, mesmo em centros especializados.

Geralmente estão associadas a outras condições e doenças, as mais marcantes são alcoolismo, desnutrição e pós-transplante renal; podendo ocorrer, de maneira mais rara, após procedimentos cirúrgicos, em estados como polidipsia psicogênica, como manifestação de doença de Wilson ou doença celíaca e também em insuficiência adrenal primária. (DAGUR G e KHAN AS, 2015; WU WJ, et al., 2014; VERMA R e RAI D, 2013; MARTIN RJ, 2004; AGUIAR AC, et al. 2004). Este artigo tem por objetivo detalhar o caso de uma paciente que evoluiu com mielinólise extrapontina (MEP) secundária à hipernatremia, confirmada por ressonância magnética, com desfecho favorável.

\section{DETALHAMENTO DO CASO}

Paciente de 30 anos de idade, sexo feminino, natural e residente em Belém, estado do Pará, sem antecedentes mórbidos pessoais prévios, começou a apresentar, em maio de 2018, quadro de cefaleia 
temporal à direita, sede excessiva e polaciúria associadas à prurido ocular e fotofobia. Procurou atendimento médico devido ao quadro, levantado o diagnóstico de migrânea e iniciado cefalium para controle.

Após 2 meses de tratamento e sem regressão sintomática, paciente procurou novamente o serviço de saúde. Na ocasião, solicitada tomografia e ressonância magnética de crânio, ambas sem contraste, além de exames laboratoriais.

A paciente não conseguiu realizar os exames de imagem, mas os exames laboratoriais daquela ocasião já demonstravam hipernatremia (sódio de $151 \mathrm{mEq} / \mathrm{L}$ ). Com a progressão dos sintomas, a paciente começou a apresentar disartria, disfagia, fraqueza muscular e engasgos frequentes.

Dia 3 de outubro de 2018, 5 meses após início dos sintomas, apresentou quadro de engasgo grave enquanto almoçava, com desconforto respiratório intenso, e foi levada à unidade de pronto atendimento (UPA), onde foi intubada de imediato e, nos exames iniciais, detectado broncoaspiração de conteúdo. Permaneceu internada por 7 dias em UPA até ser transferida a um Hospital no dia 10 de outubro de 2018.

Durante internação no Hospital, apresentou inicialmente tetraparesia hiporreflexa, com força muscular grau 1 globalmente, e passou a desenvolver episódios convulsivos sem manifestações motoras (estado epiléptico não convulsivo).

Exames laboratoriais iniciais evidenciaram hipernatremia de $180 \mathrm{mEq} / \mathrm{L}$. Paciente foi conduzida com anticonvulsivantes e correção de hipernatremia com soro fisiológico a $0,45 \%$. A curva de sódio do início da internação até normalização está registrada no Gráfico 1. No dia 16 de outubro de 2018, realizou uma ressonância magnética de crânio, evidenciando mielinólise extrapontina (Figuras 1a, 1b e 1c).

Gráfico 1 - Níveis de sódio sérico, em mEq/L, do início da internação até a normalização.

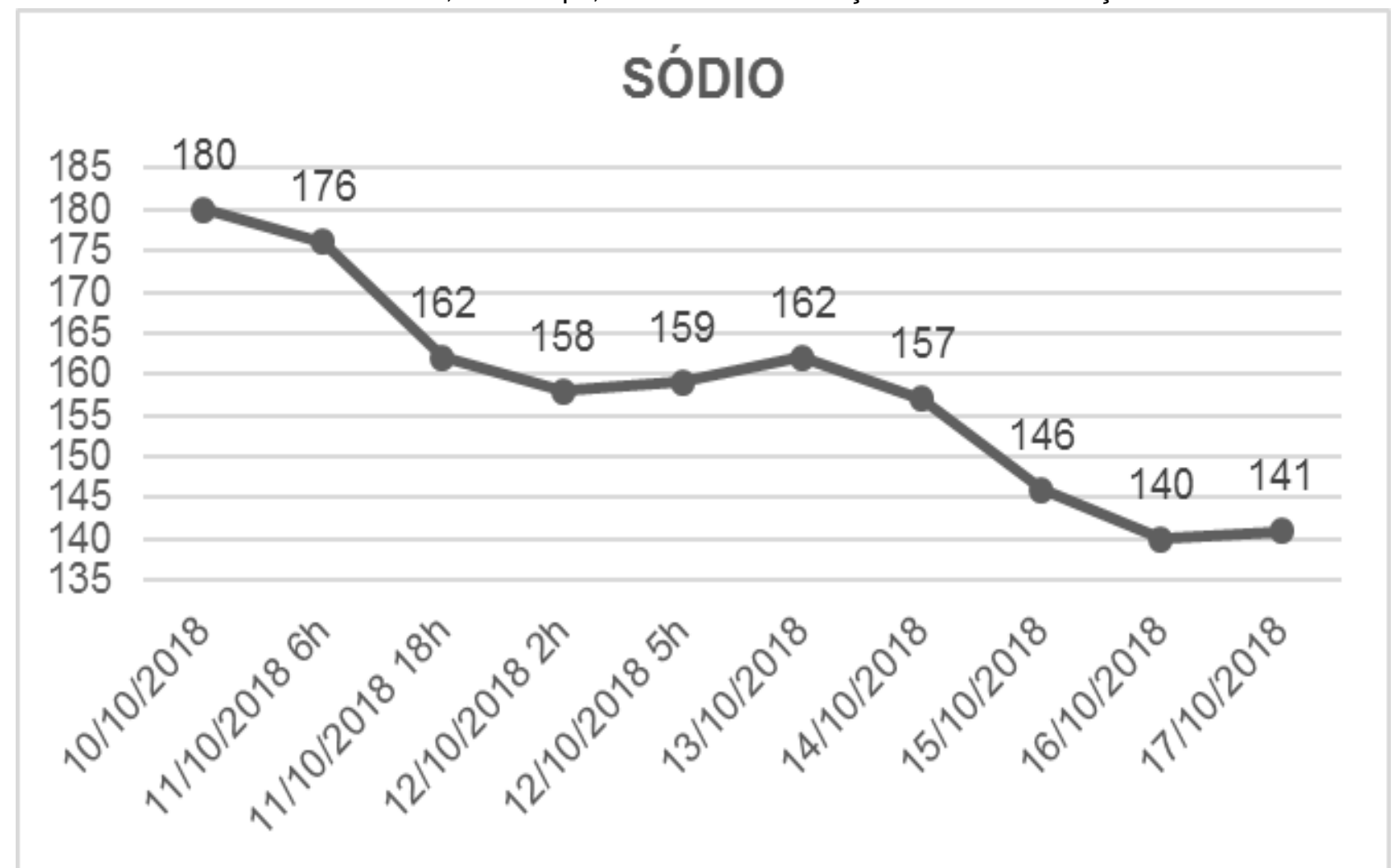

Legenda: Resultados de exames laboratoriais da paciente do início da internação até a normalização. Notase a redução gradual dos níveis de sódio ao longo dos dias, demorando 7 dias até sua normalização. Após o dia 17/10/2018, a paciente não apresentou novas grandes oscilações nos níveis de sódio.

Fonte: Rosa Neto A, Kahwage CB, Kahwage Neto SG; 2019. 
Figura 1 - Imagens da primeira ressonância magnética do crânio realizada durante a internação.
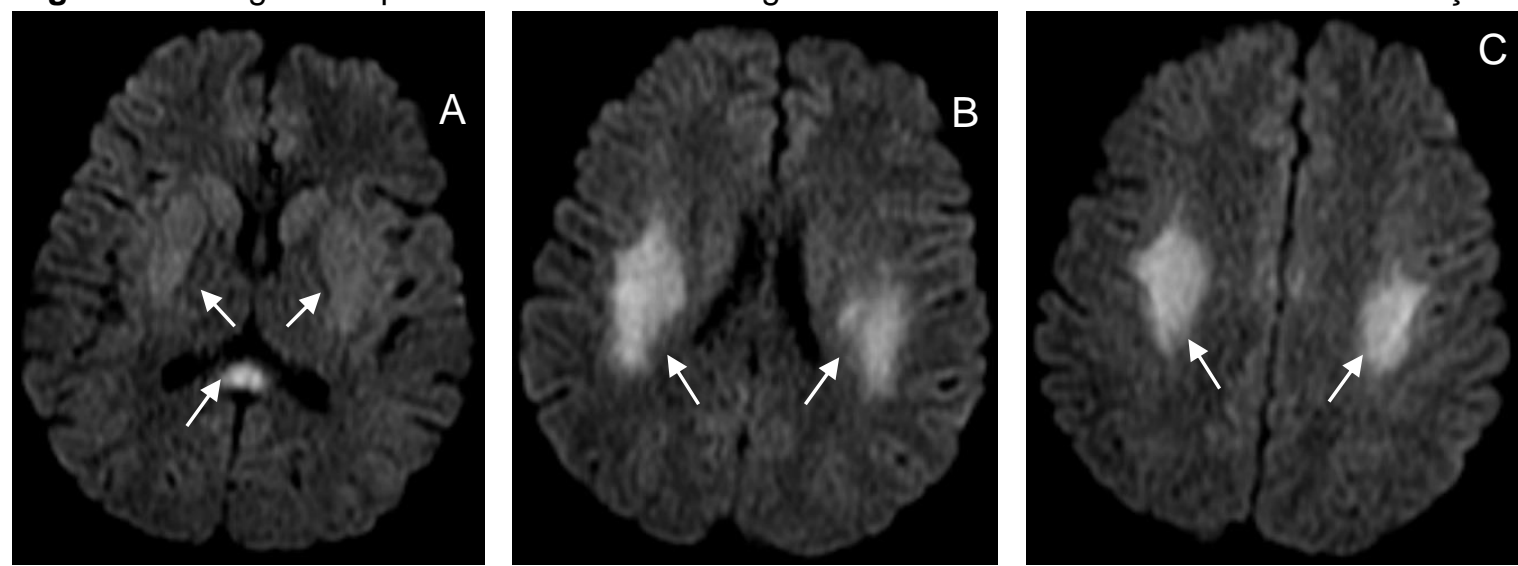

Legenda: Figura 1A Axial Difusão: Restrição no esplênio do corpo caloso e núcleos da base. Figuras 1B e 1C Axial Difusão: Restrição no centro semioval relacionado a edema vasogênico nos tratos córticoespinhais. Fonte: Rosa Neto A, Kahwage CB, Kahwage Neto SG; 2019.

$\mathrm{Na}$ internação em Unidade de Terapia Intensiva (UTI), apresentou desmame ventilatório difícil, com diversas falhas no protocolo de extubação institucional, necessitando confecção de traqueostomia precoce. Neste período, também foi realizada investigação para outras doenças infecciosas do sistema nervoso central devido a progressão lenta da melhora de força motora, com coleta de líquor em dois momentos, ambos com culturas, reação em cadeia de polimerase (PCR), e sorologias negativas para vírus, bactérias e fungos. Realizou nova ressonância magnética de crânio de controle (dia 23/10/2018) que já não mais evidenciava as lesões apresentadas anteriormente.

Ao longo dos dias, apresentou melhora dos níveis natrêmicos, com recuperação dos movimentos dos membros e sem novos episódios de crises convulsivas após ajustes de drogas anticonvulsivantes. Voltou a deambular no dia 2 de novembro de 2018. No dia 5 de novembro de 2018, recebeu alta da UTI com cânula de traqueostomia, já com força muscular grau 3 globalmente e sem novos episódios convulsivos. Apresentava apenas dor neuropática em membros inferiores, mais forte no membro inferior direito.

$\mathrm{Na}$ enfermaria permaneceu por 21 dias devido dificuldade de decanulação de traqueostomia. A dor neuropática foi melhorada com doses otimizadas de amitriptilina e pregabalina. Após intensificação de fisioterapia, com melhora clínica e decanulação sem intercorrências, teve alta hospitalar no dia 26 de novembro de 2018 com força muscular grau 4 globalmente.

Atualmente, encontra-se em acompanhamento no serviço de clínica médica do hospital. Deambula com auxílio de bengala do lado direito, onde tem menor força no membro inferior. Permanece com quadro de disfagia leve para sólidos e está em acompanhamento com fonoaudiologia, com melhora progressiva deste sintoma. A dor neuropática em membros inferiores está controlada com o uso de gabapentina e amitriptilina. Apresentou 3 episódios convulsivos em julho de 2019 por falta de carbamazepina; após aquisição do medicamento e tomadas regulares, não teve novas convulsões. Os níveis de sódio permanecem controlados, sendo os níveis ambulatoriais variáveis entre 140 e $146 \mathrm{mEq} / \mathrm{L}$.

\section{DISCUSSÃO}

Diversos componentes fazem parte do controle de volume cerebral, sendo os principais classificados em dois grupos: os neurotransmissores e os eletrólitos. Dentre os neurotransmissores, os principais são glutamina, glutamato, taurina e mioinositol e quando ocorre uma variação na quantidade deles, há modificação dos potenciais de ação das membranas, aumentando a chance de convulsão.

Em relação aos eletrólitos, o principal envolvido nesta regulação é o sódio. Ele é responsável pela osmolaridade plasmática e cerebral, sendo suas variações diretamente relacionadas com esta variável (VERBALIS JG, 2006). Entretanto, não somente os níveis de sódio estão envolvidos na sintomatologia dos 
pacientes; o mais importante é a velocidade de instalação do distúrbio. Quanto mais rápida a variação de sódio no organismo, maior a chance de desequilíbrio osmótico e desenvolvimento de alterações relacionadas à edema ou desidratação celular, o que impacta na sintomatologia e mortalidade geral (VERBALIS JG, 2006). Por outro lado, quando a variação ocorre lentamente, há um mecanismo adaptativo em relação ao volume celular e quantidade eletrolítica, consequentemente os pacientes são oligossintomáticos ou assintomáticos. Isto explica como ocorrem as lesões decorrentes de variações nos níveis de sódio em pacientes hiponatrêmicos, hipernatrêmicos e até em pacientes com níveis normais de sódio (GERMINIANI FMB, et al., 2002). Até o momento, não se sabe qual o valor exato de variação para que ocorram as síndromes de desmielinização. Aceita-se que 8-10 mEq em 24 horas seja uma taxa adequada com base em estudos feitos em animais (MARTIN RJ, 2004).

Os principais sintomas relacionados às síndromes são tremor, quadriplegia, rebaixamento de nível de consciência, dificuldade de deglutição e fraqueza muscular, porém, há uma outra gama de sintomas neurológicos que podem estar relacionados, a depender de comorbidades e do local afetado. A mortalidade é alta e o desfecho imprevisível, podendo apresentar recuperação completa dos sintomas, recuperação parcial ou até levar a óbito (COÇA KL, et al., 2017; KHAN AS e DAGUR G, 2015; JURNO ME, et. al., 2012; BRITO AR, et al., 2006).

O tratamento específico para as síndromes ainda está em seus primeiros passos. Murthy SB, et al. (2013), descreveu 3 casos com administração de imunoglobulina intravenosa, com melhora após 3 dias de administração do medicamento. Saner FH (2008), submeteu seu paciente transplantado renal ao tratamento com imunoglobulina e plasmaférese, obtendo bons resultados após 6 dias. Apesar de mecanismo ainda não completamente esclarecido, acredita-se que estas substâncias sejam responsáveis pela modulação e diminuição da atividade imune de citocinas lançadas no sistema nervoso central após quebra da barreira hematoencefálica (DAGUR, G e KHAN SA, 2015). Neste sentido, o principal subsídio é a prevenção, fazendo a correção de sódio em níveis adequados e mantendo um bom monitoramento da correção. Aos que se recuperam do acometimento inicial da doença, há a reabilitação com fisioterapia e fonoaudiologia, visando reversão e minimização de sequelas e melhora da qualidade de vida (COÇA KL, et al., 2017; MARTIN RJ, 2004).

Chang KY, et al. (2014), em seu trabalho, estudou uma paciente de 40 anos que se apresentou com quadro inicial de fraqueza muscular, vômitos e vertigem por 2 meses. Após correção de distúrbio ácido-base com bicarbonato, a paciente evoluiu de $142 \mathrm{mEq} / \mathrm{L}$ para $172 \mathrm{mEq} / \mathrm{L}$ de sódio em um dia. Começou a apresentar disartria, dificuldade de deglutição e tetraparesia. Foi diagnosticada com mielinólise pontina central através de ressonância magnética de crânio. Através de plasmaférese, a paciente obteve rápida melhora sintomática. Hezagi MO e Mashankar A (2013) descreveram outro caso de mielinólise pontina central associada a um quadro de estado hiperosmolar hiperglicêmico; a paciente também se apresentava inicialmente com quadro de letargia, porém, sem sinais neurológicos focais. Teve recuperação total mais prolongada, ao longo de quatro semanas, sem tratamento específico para hipernatremia, apenas com normalização dos distúrbios eletrolíticos e correção de glicemia.

Levin J, et. al. (2012) estudaram uma paciente de 73 anos com alterações de nível de consciência associada a sódio sérico de $178 \mathrm{mEq} / \mathrm{L}$, que foi corrigido ao longo de sete dias. A tomografia de crânio não mostrou anormalidades, porém, no décimo segundo dia de admissão foi aventada a hipótese de mielinólise pontina devido coma prolongado mesmo com a paciente sem sedativos. Solicitada ressonância do crânio que mostrou mielinólise pontina e extrapontina. Apresentou melhora radiológica com nova ressonância do crânio feita 14 dias após a primeira.

Nenhuma terapêutica específica para hipernatremia foi empregada e a paciente recebeu alta após 33 dias de internação hospitalar. A paciente do caso deu entrada no serviço proveniente de outro hospital, onde passou 7 dias. Inicialmente apresentava dificuldade de deglutição e fraqueza muscular como principais sintomas, de acordo com as características descritas na literatura. Após, também passou a desenvolver quadros convulsivos, devido desregulação relacionada aos neurotransmissores, e tetraparesia, também de acordo com as principais manifestações encontradas na literatura (DAGUR G e KHAN SA, 2015; CHANG KY, et al., 2014; HEZAGI MO e MASHANKAR A, 2013). 
A primeira ressonância magnética realizada evidenciou lesões no trato córtico-espinhal e núcleos da base, que estão de acordo com o quadro clínico da paciente de tetraparesia hiporreflexa. Já a segunda ressonância, realizada sete dias após a primeira, não apresentou alterações, com regressão radiológica rápida. Levin $\mathrm{J}$, et al. (2012), descreveu um caso no qual a paciente apresentou melhora radiológica importante em 14 dias, porém não há registros na literatura médica de quanto tempo é necessário para recuperação das lesões encefálicas após sua instalação. Seus níveis de sódio iniciais eram de $180 \mathrm{mEq} / \mathrm{L}$. Após correção do distúrbio eletrolítico ao longo dos dias, ela apresentou melhora do quadro clínico, com regressão parcial ao final do tratamento hospitalar. Contudo, a despeito das evoluções favoráveis, nem todos os casos apresentam regressão sintomática, havendo desfechos catastróficos com óbito registrados na literatura (BRITO AR, et al., 2006).

Nenhum tratamento específico para hipernatremia foi realizado na paciente além da correção do distúrbio. Reforça-se a necessidade de ao realizar correções hidroeletrolíticas, estas sejam feitas de forma rigorosa, observando-se o quadro clínico dos pacientes, pois a melhor forma a se evitar lesões intracranianas ainda é a preventiva. Muito ainda se tem a aprender a respeito das individualidades relacionadas às síndromes de desmielinização osmótica. Quando estas ocorrem, não é possível prever o desfecho, podendo ser totalmente favorável ou catastrófico, a principal medida a ser tomada ainda é a preventiva, com precaução ao corrigir distúrbios eletrolíticos, atentando para manifestações clínicas. E na sua suspeita um estudo aprofundado deve ser conduzido a fim de aumentarem os diagnósticos precisos dessas enfermidades.

\section{REFERÊNCIAS}

1. ADAMS RD, et al. Central pontine myelinolysis: A hitherto undescribed disease occurring in alcoholic and malnourished patients. Archives of neurology and psychiatry, 1959; 81(2):154-172.

2. AGUIAR AC, et al. Mielinólise pontina e extrapontina associada a shoshin beribéri em paciente etilista. Arquivos de Psiquiatria, 2004; 62 (3A): 733-736.

3. BRITO AR, et. al. Central pontine and extrapontine myelinolysis: report of a case with tragic outcome. Jornal de Pediatria, 2006; 82(2): 157-160.

4. CHANG KY, et. al. Plasma exchange successfully treats central pontine myelinolysis after acute hypernatremia from intravenous sodium bicarbonate therapy. BMC Nephrology, 2014. 15(56).

5. COÇA KL, et. al. Achados de fala, deglutição e qualidade de vida na mielinólise extrapontina: relato de caso de uma adolescente com germinoma do sistema nervoso central. Revista CEFAC, 2017; 19(1): 135-140.

6. GERMINIANI FMB, et al. Mielinólise pontinha central e extrapontina em paciente alcoolista sem distúrbios hidroeletrolíticos. Arquivo neuropsiquiatria, 2002; 60(4): 1030-1033.

7. HEZAGI MO, MASHANKAR A. Central pontine myelinolysis in the hyperosmolar hyperglycaemic state. Medical principles and practice, 2013. 22: 96-99.

8. JURNO ME, et al. Síndrome de desmielinização osmótica: relato de caso com evolução favorável. Radiologia Brasileira, 2012; (45)1: 61-62.

9. ISMAIL FY, et al., Clinical semiology and neuroradiologic correlates of acute hypernatremic osmotic challenge in adults: a literature review. American Journal of Neuroradiology, 2013; 34: 2225-32.

10. KHAN AS, DAGUR G. Current concepts in pontine myelinolysis: review of literature. Translational biomedicine, 2015; (6) 4: 1-7.

11. LAUBENBERGER J, et al., Central pontine myelinolysis: clinical presentation and radiologic findings. European Radiology, 1996; 6(2): 177-183.

12. LEVIN J, et al. Pontine and extrapontine myelinolysis associated with hypernatremia. Clinical neurology and neurosurgery, 2012. 1290-1291.

13. MARTIN RJ. Central pontine and extra-pontine myelinolysis: The osmotic demyelination syndromes. Journal of Neurology, Neurosurgery and Psychiatry, 2004; (75) suppl III: 22-28.

14. MURTHY SB, et. al. Osmotic demyelination syndrome: variable clinical and radiologic response to intravenous immunoglobulin therapy. Neurological Sciences, 2013; (34)4:581-584.

15. SANER FH, et al. Treatment of central pontine myelinolysis with plasmapheresis and immunoglobulin in liver transplant patient. Transplant International, 2008; (21)4:390-391.

16. VERBALIS JG. Control of brain volume during hypoosmolality and hyperosmolality. Advances in Experimental Medicine and Biology, 2006. (576):113-129.

17. VERMA R, RAI D. Central pontine myelinolysis associated with Wilson disease in a 7-year-old child. BMJ Case Report (online), 2013.

18. WU JW, et al. Extrapontine myelinolysis in a patient with primary adrenal insufficiency. Acta Neurologica Taiwan, $2014 ; 23(4): 146-152$. 